\title{
Studies on the Biosynthesis of Some Furocoumarins Present in Ruta graveolens
}

\author{
G. Caporale, F. Dall'Acqua, A. Capozzi, S. Marciani, and R. Crocco
}

Institute of Pharmaceutical Chemistry of the University, Centro di Chimica del Farmaco e dei Prodotti biologicamente attivi del Consiglio Nazionale delle Ricerche, Padua (Italy)

(Z. Naturforsch. 26 b, 1256-1259 [1971] ; received August 26, 1971, revised September 23, 1971)

The biosynthesis of some furocoumarins present in Ruta graveolens has been studied administering labeled psoralen, xanthotoxin, rutaretin, marmesin and 7-hydroxycoumarin-derivatives.

The results obtained indicate that the biosynthetic pathway involves not only psoralen, but also xanthotoxin and bergapten, by means of methoxylation or demethoxylation reactions.

Moreover results achieved from administering rutaretin demonstrate that this substance is a new very effective natural precursor for xanthotoxin; in a parallel way marmesin is incorporated into psoralen. The reported results indicate that also at the stage of natural $4^{\prime}, 5^{\prime}$-dihydrofurocoumarins intermediates an interconversion can occur.

Finally the essential role of umbelliferone in the biosynthesis of furocoumarins has been confirmed.

The common strain Ruta graveolens L., contains many different furocoumarin-derivatives, located primarily in the leaves ${ }^{1-10}$.

It is well known that these substances show very interesting photobiological properties, which explain the photodermatites ${ }^{11}$ that this plant can provoke. The biological properties of furocoumarins have been widely investigated in our Institute over a long period $^{12}$ and their molecular mechanism of photosensitization has been clarified ${ }^{13,14}$.

Many Authors ${ }^{15-23}$ have investigated the biosynthetic pathway of furocoumarins and the results obtained with different plants and herbs indicated that the biosynthesis of this substances involves in a first stage the formation, from the corresponding oxygenated cinnamic acid, of 7-hydroxycoumarin-derivative.

Request for reprints should be sent to Dr. G. CAPorale, Universitá di Padova, Istituto di Chimica Farmaceutica, Via Marzolo 5, 35100 Padova (Italien).

1 W. BrandT, C. II, 1199 [1915].

2 H. Mühlemann, Pharmac. Acta Helvetiae 13, 45 [1938].

3 G. Rodighiero, G. Caporale, and G. Albiero, Gazz. chim. ital. 84, 874 [1954].

4 I. Novak, G. Buzas, E. Minker, M. Koltai, and K. Szendrei, Planta med. [Stuttgart] 14, 151 [1966].

5 I. Novak, K. Szendrei, G. Buzas, E. Minker, and M. KoltaI, Acta pharm. Hung. 37, 225 [1967].

${ }^{6}$ J. Reisch, I. Novak, K. Szendrei, and E. Minker, Pharmacie 21, 628 [1966].

7 J. Reisch, I. Novak, K. Szendrei, and E. Minker, Acta pharm. Suecica 4, 179 [1967].

8 J. Reisch, K. Szendrei, E. Minker, and I. Novak, Tetrahedron Letters [London] 41, 4395 [1968].

9 G. Schneider, H. Müller, and P. Pfaender, Arch. Pharmaz. 300, 73 [1967].

10 G. Schneider and H. Müller, Arch. Pharmaz. 300, 913 [1967].

11 H. KUSKe, Arch. Dermatologie u. Syphilis 178, 112 [1938].
This represents a first step in the biosynthesis of furocoumarins and in particular umbelliferone (or its glucoside skimmin) has been proposed as the physiological precursor in this first stage ${ }^{16,19}$.

Successively the formation of the furan ring occurs on the 7-hydroxycoumarin-derivative through an isoprenylation reaction which leads to a $4^{\prime}, 5^{\prime}$-dihydrofurocoumarin-derivative ${ }^{15,16}$. Recently STECK and BROWN ${ }^{17}, 19$ suggested that the natural intermediate which is involved in this stage is marmesin (5), [4', $5^{\prime}$-dihydro-5' - (1-hydroxy-1-methylethyl) furocoumarin].

These Authors implied that this substance is subsequently transformed in psoralen; finally at this stage hydroxylation and $\mathrm{O}$-alkylation reactions occur on psoralen (1) and consequently the other furocoumarins, such as bergapten (2), xanthotoxin (3)

12 L. Musajo, G. Rodighiero, and G. Caporale, Bull. Soc. chim. biol. 36, 1213 [1954].

13 L. Musajo, G. Rodighiero, and F. Dall'Acqua, Experientia [Basel] 21, 24 [1965].

14 L. Musajo and G. Rodighiero, Photochem. Photobiol. 11, 27 [1970].

15 H. G. Floss and U. Mothes, Phytochem. 5, 161 [1966].

16 H. G. Floss and H. Paikert, Phytochem. 8, 589 [1969].

17 W. Steck, M. El-Dakhakhny, and S. A. Brown, Tetrahedron Letters [London] 54, 4805 [1969].

18 S. A. Brown, Phytochem. 9, 2471 [1970].

19 S. A. Brown, M. El-DakhaKhnY, and W.Steck, Canad. J. Biochem. 48, 863 [1970].

20 W. Steck and S. A. Brown, Canad. J. Biochem. 48, 872 [1970].

21 G. Caporale, A. Breccia, and G. Rodighiero, Prepn. Bio-Med. Appl. Labeled Mol., Proc. Sympos., pag. 103 , Venice 1964.

22 G. Caporale, F. Dall'Acqua, S. Marciani, and A. CA POZZI, Z. Naturforsch. 25 b, 700 [1970].

23 G. Caporale, F. Dall'Acqua, S. Marciani Magno, and A. CAPOZZI, Herba Hungarica, 1971, in press. 
and so on, can be formed.

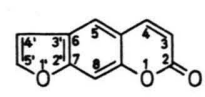

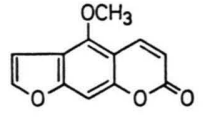

2

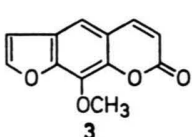

3
Fig. l.

In a recent paper experimenting with Ruta graveolens ${ }^{23}$ we found that other $4^{\prime}, 5^{\prime}$-dihydroderivatives, such as $4^{\prime}, 5^{\prime}$-dihydropsoralen, $4^{\prime}, 5^{\prime}$-dihydrobergapten and also $4^{\prime}, 5^{\prime}$-dihydroxanthotoxin can act as possible precursors for the corresponding furocoumarins; moreover we obtained results which suggested that a transformation of one of these furocoumarins occurred in the other two, probably through methoxylation or demethoxylation reactions.

With the aim of confirming this possibility of interconversion among the various substances contained in Ruta graveolens, we have administered labeled psoralen (1) and xanthotoxin (3) to the plant and then followed their possible transformation in other furocoumarins.<smiles>CC(C)(O)C1Cc2cc3ccc(=O)oc3c(O)c2O1</smiles><smiles>CC(C)(O)C1Cc2cc3ccc(=O)oc3cc2O1</smiles>

Fig. 2.

Moreover we have also fed Ruta graveolens with labeled rutaretin (4), [4', $5^{\prime}$-dihydro-5'-(1-hydroxy1-methylethyl) -8-hydroxy-furocoumarin]; this substance, which is present in the herb ${ }^{9}$, contains an hydroxyl-group in -8-position and consequently would be a precursor for xanthotoxin. We have tested this substance and for comparison also marmesin (5) to establish if in Ruta graveolens there exists a different physiological precursor other than marmesin.

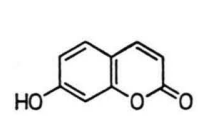

6

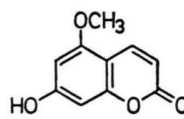

7

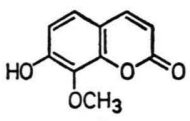

8
Fig. 3.

24 H. V. Pechmann, Chem. Ber. 17, 929 [1884].

$25 \mathrm{G}$. Rodighiero and C. Antonello, Farmaco [Pavia] 10, 889 [1955].

26 G. Rodighiero and C. Antonello, Ann. Chimica 46, 960 [1956].
Finally we have studied the role in this herb of labeled 5-methoxy-7-hydroxycoumarin (7) and 7-hydroxy-8-methoxycoumarin (8) and also umbelliferone (6).

\section{Materials and Methods}

\section{Preparation of labeled substances}

7-hydroxycoumarin or umbelliferone $(6)^{24}, 5$-methoxy-7-hydroxycoumarin (7) ${ }^{25}$ and 7-hydroxy-8-methoxycoumarin (8) ${ }^{26}$ have been prepared by synthesis in this Institute.

Marmesin (5) ${ }^{27}$ (extracted from Aegle marmelos, Correa) and rutaretin (4) ${ }^{9}$ (extracted from Ruta graveolens) have kindly been provided by Prof. Dr. A. Chatterjee and Prof. Dr. G. Schneider respectively.

All these substances have been tritiated with the Wilzb a c h method ${ }^{28}$.

Purification of these substances has been carried out as described elsewhere ${ }^{22}$. Specific radioactivity of these compounds is reported in Table I, II and III.

\section{Feeding Ruta graveolens with the labeled compounds}

Aqueous solutions of the various labeled substances added to an equal volume of an "aqueous nutritious solution" 29 were introduced separately into $250 \mathrm{ml}$ beakers containing seven months old herbs, which were immersed in these feeding solutions.

"Metabolism time" lasted seven days and during this period "aqueous nutritious solution" was added to maintain constant the level of the liquid layer.

During this "metabolism period" the herbs were illuminated with $500 \mathrm{~W}$ OSRAM HWL lamps. Control experiments were performed under the same experimental conditions without feeding labeled compounds.

\section{Isolation and purification of furocoumarins}

All the herbs, which were fed with the same labeled compound, were collected together after the "metabolism time", dried, completely triturated and extracted in an extraction apparatus; the methanolic extract after concentration was treated up following the procedure for preparing the "coumarinic extract" as described elsewhere ${ }^{21,22,30}$.

Psoralen, bergapten and xanthotoxin, which are commonly considered the most important furocoumarins contained in this herb ${ }^{19,23}$, have been isolated and purified as follows: in a first stage a initial separation of the three substances was performed using thin layer preparative chromatoplates (Merck Cat. 5717). $1 \mathrm{ml}$ of

27 A. Chatterjee and S. S. Mitra, J. Amer. chem. Soc. 71, 606 [1949].

28 K. E. Wilzbach, J. Amer. chem. Soc. 79, 1013 [1957].

29 E. J. HewitT, Plant Physiology, vol. 3, pag. 101, Academic Press, New York 1963.

30 E. SpÄтH, Chem. Ber. 70 A, 83 [1937]. 
"coumarinic extract" was applied as a band on every plate and this was developed with a mixture of cyclohexane-ethyl acetate $(2: 1, v / v)$. In this way the traces of administered labeled substances were separated from the three furocoumarins. In a second step the area corresponding to the three furocoumarins was eluted with ethyl alcohol (using $25 \mathrm{ml}$ of solvent for every plate) and the obtained solutions were filtered through a Millipore Mitef SM $5 \mu$ membrane; after concentration they were applied to 3 chromatopapers Whatman $3 \mathrm{MM}$ which were developed with water. In this way psoralen and xanthotoxin were separated from bergapten. In a third stage silica gel analytical chromatoplates (Merck Cat. 5715) were used to isolate psoralen from xanthotoxin, developing plates with chloroform. The radiochemical purity of psoralen, bergapten and xanthotoxin was tested on silica gel thin layer chromatoplates using cyclohexane-ethyl acetate $(2: 1, v / v)$ as a solvent system. The amounts of these three substances have been measured spectrophotometrically, determining the O.D. of the corresponding solutions obtained by elution from the above mentioned chromatoplates (the bands of the three furocoumarins were brought into evidence by their fluorescence when exposed at $365 \mathrm{~nm}$ light).

Spectrophotometrical determinations and radiochemical measurements

Spectrophotometrical determinations were performed in a parallel way both recording the ultraviolet spectra of the ethanolic solutions of the three furocoumarins using a 124 double beam model Perkin-Elmer spectrophotometer and successively by reading the absorbance at $\lambda$ max by means of a single beam CF4 OPTICA instrument. Radiochemical measurements were performed by a liquid scintillator spectrometer Beckman
LS 150, using a dioxane base liquid scintillator: naphthalene g 120, 2,5-diphenyl-oxazole g 4, 2,2'-p-phenylenbis (5-phenyl-oxazole) g 0.075 in dioxane up to $1000 \mathrm{ml}$ of solution. The apparatus efficiency in the experimental conditions used was $30-34 \%$ for tritium.

\section{Results and Discussion}

The results obtained feeding Ruta graveolens with psoralen (1) and xanthotoxin (3) are reported in Table I.

These data indicate that feeding experiments with psoralen lead to a good incorporation of radioactivity both into bergapten and also into xantho. toxin and moreover similar experiments with xanthotoxin show that this substance acts as an effective precursor for psoralen and bergapten. These results clearly demonstrate the possibility of interconversion by means of methoxylation or demethoxylation reactions. This new aspect indicates that the biosynthetic pathway of furocoumarins in Ruta graveolens cannot necessarily involve only psoralen, as previously reported ${ }^{19}$, but also xanthotoxin and bergapten.

The results obtained feeding the herb with rutaretin (4), and for comparison also with marmesin (5), are reported in Table II. Administered rutaretin leads mainly to a very high incorporation of radioactivity into xanthotoxin; marmesin, similarly tested, leads essentially to a strongly evident incorporation of radioactivity into psoralen. As dilution

\begin{tabular}{|c|c|c|c|c|c|c|c|}
\hline \multirow{2}{*}{$\begin{array}{l}\text { Compound } \\
\text { administered }\end{array}$} & \multirow{2}{*}{$\begin{array}{l}\text { Specific } \\
\text { activity } \\
{[\mathrm{dpm} / \mu \text { moles }} \\
\left.\times 10^{6}\right]\end{array}$} & \multicolumn{2}{|l|}{ Psoralen } & \multicolumn{2}{|l|}{ Bergapten } & \multicolumn{2}{|l|}{ Xanthotoxin } \\
\hline & & $\begin{array}{l}\text { Specific } \\
\text { activity } \\
{[\mathrm{dpm} / \mu \mathrm{moles}]}\end{array}$ & dilution* & $\begin{array}{l}\text { Specific } \\
\text { activity } \\
{[\mathrm{dpm} / \mu \text { moles }]}\end{array}$ & dilution* & $\begin{array}{l}\text { Specific } \\
\text { activity } \\
{[\mathrm{dpm} / \mu \text { moles }]}\end{array}$ & dilution* \\
\hline Psoralen & 7.07 & 319,000 & 23.3 & 46,850 & 151 & 7790 & 908 \\
\hline Xanthotoxin & 1.257 & 2700 & 465 & 4040 & 321 & 50,000 & 25.1 \\
\hline
\end{tabular}

Table I. Conversion of psoralen and xanthotoxin to other furocoumarins. * Dilution is defined as the ratio between the specific activity of compounds administered and that of isolated furocoumarins.

\begin{tabular}{|c|c|c|c|c|c|c|c|}
\hline \multirow{2}{*}{$\begin{array}{l}\text { Compound } \\
\text { administered }\end{array}$} & \multirow{2}{*}{$\begin{array}{l}\text { Specific } \\
\text { activity } \\
{[\mathrm{dpm} / \mu \text { moles }]} \\
\times 10^{6}\end{array}$} & \multicolumn{2}{|l|}{ Psoralen } & \multicolumn{2}{|l|}{ Bergapten } & \multicolumn{2}{|l|}{ Xanthotoxin } \\
\hline & & $\begin{array}{l}\text { Specific } \\
\text { activity } \\
{[\mathrm{dpm} / \mu \text { moles }]}\end{array}$ & dilution* & $\begin{array}{l}\text { Specific } \\
\text { activity } \\
{[\mathrm{dpm} / \mu \text { moles }]}\end{array}$ & dilution* & $\begin{array}{l}\text { Specific } \\
\text { activity } \\
{[\mathrm{dpm} / \mu \text { moles }]}\end{array}$ & dilution* \\
\hline Rutaretin & 16.15 & 4050 & 3990 & 6800 & 2700 & 170,000 & 95 \\
\hline Marmesin & 5.00 & 53,000 & 94.4 & 8380 & 597 & 1767 & 2830 \\
\hline
\end{tabular}

Table II. Conversion of rutaretin and marmesin into furocoumarins. * Dilution is defined as the ratio between the specific activity of compounds administered and that of isolated furocoumarins. 


\begin{tabular}{|c|c|c|c|c|c|c|c|}
\hline \multirow{2}{*}{$\begin{array}{l}\text { Compound } \\
\text { administered }\end{array}$} & \multirow{2}{*}{$\begin{array}{l}\text { Specific } \\
\text { activity } \\
{[\mathrm{dpm} / \mu \text { moles }]} \\
\times 10^{6}\end{array}$} & \multicolumn{2}{|l|}{ Psoralen } & \multicolumn{2}{|l|}{ Bergapten } & \multicolumn{2}{|l|}{ Xanthotoxin } \\
\hline & & $\begin{array}{l}\text { Specific } \\
\text { activity } \\
{[\mathrm{dpm} / \mu \text { moles }]}\end{array}$ & dilution* & $\begin{array}{l}\text { Specific } \\
\text { activity } \\
{[\mathrm{dpm} / \mu \text { moles }]}\end{array}$ & dilution* & $\begin{array}{l}\text { Specific } \\
\text { activity } \\
{[\mathrm{dpm} / \mu \text { moles }]}\end{array}$ & dilution* \\
\hline $\begin{array}{l}\text { 7-hydroxy- } \\
\text { coumarin }\end{array}$ & 11.24 & 81,500 & 138 & 24,900 & 451 & 7190 & 1562 \\
\hline $\begin{array}{l}\text { 5-methoxy-7- } \\
\text { hydroxy-coumarin }\end{array}$ & $\begin{array}{l}23.3 \\
\text { in }\end{array}$ & 1170 & 19,900 & 22,650 & 1030 & 680 & 34,200 \\
\hline $\begin{array}{l}\text { 7-hydroxy-8- } \\
\text { methoxy-coumari }\end{array}$ & in 2.92 & 1365 & 2540 & 587 & 4970 & 248 & 11,750 \\
\hline
\end{tabular}

Table III. Conversion of oxygenated coumarins into furocoumarins. * Dilution is defined as the ratio between the specific activity of compounds administered and that of isolated furocoumarins.

values reported in Table II indicate, the two substances are incorporated with the same effectiveness. On the basis of these data we can conclude that other than marmesin, as previously reported ${ }^{19}$, another new physiological intermediate, rutaretin, can be involved in the biosynthetic pathway of furocoumarins in this herb. Moreover we observe that from these two precursors have been obtained not only the corresponding furocoumarins, but also the other two examined acquired definite amounts of radioactivity, confirming the possibility of interconversion.

Finally 5-methoxy-7-hydroxycoumarin (7) and 7-hydroxy-8-methoxycoumarin (8) are incorporated only in a very small degree into psoralen, bergapten and xanthotoxin as indicated in Table III, while umbelliferone (6) leads to a very high incorporation of radioactivity into psoralen, and also, in decreasing amounts, into bergapten and into xanthotoxin.

These data confirm the previously obtained results by other Authors and indicate that umbelliferone is a good natural precursor in the biosynthesis of furocoumarins. Moreover is confirmed that in Ruta graveolens, at the stage of 7-hydroxycoumarin-derivative, methoxylation or hydroxylation reactions cannot occur.

Considering the various intermediates involved in the two first stages in the biosynthetic pathway of furocoumarins in Ruta graveolens we found only umbelliferone at stage of 7-hydroxycoumarin-derivative and rutaretin and marmesin at the level of $4^{\prime}, 5^{\prime}$-dihydrofurocoumarin-derivatives. It is well known ${ }^{19}$, and also confirmed by our data, that umbelliferone cannot be hydroxylated or methoxylated and this fact agrees with the possibility that only one substance (umbelliferone) is practically involved in the biosynthesis in the first step. In contrast the fact that two intermediates, that is marmesin and rutaretin, can function at the stage of $4^{\prime}, 5^{\prime}$-dihydroderivatives, indicates that there is the possibility of hydroxylation or dehydroxylation reactions on these two substances with a consequent possible interconversion.

We are indebted to Prof. Luigr Musajo and Prof. GIOVANNI RODIGHIERo for helpful discussion on this research. 